\title{
Evaluation of the impact of atmospheric pressure loading modeling on GNSS data analysis
}

\author{
Rolf Dach · Johannes Böhm • Simon Lutz • \\ Peter Steigenberger • Gerhard Beutler
}

Received: 29 March 2010 / Accepted: 2 October 2010 / Published online: 22 October 2010

(c) Springer-Verlag 2010

\begin{abstract}
In recent years, several studies have demonstrated the sensitivity of Global Navigation Satellite System (GNSS) station time series to displacements caused by atmospheric pressure loading (APL). Different methods to take the APL effect into account are used in these studies: applying the corrections from a geophysical model on weekly mean estimates of station coordinates, using observation-level corrections during data analysis, or solving for regression factors between the station displacement and the local pressure. The Center for Orbit Determination in Europe (CODE) is one of the global analysis centers of the International GNSS Service (IGS). The current quality of the IGS products urgently asks to consider this effect in the regular processing scheme. However, the resulting requirements for an APL model are demanding with respect to quality, latency, and - regarding the reprocessing activities - availability over a long time interval (at least from 1994 onward). The APL model of Petrov and Boy (J Geophys Res 109:B03405, 2004) is widely used within the VLBI community and is evaluated in this study with respect to these criteria. The reprocessing effort of CODE provides the basis for validating the APL model. The data set is used to solve for scaling factors for each station to evaluate the geophysical atmospheric
\end{abstract}

R. Dach $(\bowtie) \cdot$ S. Lutz · G. Beutler

Astronomical Institute, University of Bern, Sidlerstrasse 5,

3012 Bern, Switzerland

e-mail: rolf.dach@aiub.unibe.ch

J. Böhm

Institute of Geodesy and Geophysics, Vienna University

of Technology, Gusshausstraße 27-29, 1040 Vienna, Austria

P. Steigenberger

Institut für Astronomische und Physikalische Geodäsie,

Technische Universität München, Arcisstraße 21,

80333 Munich, Germany non-tidal loading model. A consistent long-term validation of the model over 15 years, from 1994 to 2008 , is thus possible. The time series of 15 years allows to study seasonal variations of the scaling factors using the dense GNSS tracking network of the IGS. By interpreting the scaling factors for the stations of the IGS network, the model by Petrov and Boy (2004) is shown to meet the expectations concerning the order of magnitude of the effect at individual stations within the uncertainty given by the GNSS data processing and within the limitations due to the model itself. The repeatability of station coordinates improves by $20 \%$ when applying the effect directly on the data analysis and by $10 \%$ when applying a post-processing correction to the resulting weekly coordinates compared with a solution without taking APL into account.

Keywords Atmospheric pressure loading - GNSS processing $\cdot$ Model validation $\cdot$ Terrestrial reference frame

\section{Introduction}

The station coordinates established by the space-geodetic techniques are affected by many effects resulting in geometrical site displacements at different time scales and magnitudes. When analyzing observations of the Global Navigation Satellite Systems (GNSS) it has become widely accepted practice to apply solid Earth tides and ocean tidal loading effects using the latest models for these displacements (McCarthy and Petit 2004), thus removing the largest effects with magnitudes bigger than a few centimeters. The repeatability and consistency of weekly time series of station coordinates (e.g., within the International GNSS Service, IGS, Dow et al. 2009) are therefore well below the centimeter level-even for the vertical component 
(Ferland and Piraszewski 2009). Several other geophysical effects are currently not taken into account by the IGS for GNSS data processing - even if the expected effects amount to more than one centimeter. Depending on the location of the station these effects are crustal deformations due to, e.g., atmospheric pressure loading, ocean-induced non-tidal loading, or continental water mass surface loading in the neighborhood of the stations.

Subsequently we focus on atmospheric pressure loading (APL). Several studies successfully assessed APL for spacegeodetic data including the validation of geophysical APL models (Tregoning and van Dam 2005; Steigenberger et al. 2009a; Tesmer et al. 2008; MacMillan and Gipson 1994; van Dam and Herring 1994; Böhm et al. 2009; Bock et al. 2005, and many others). Tregoning and Watson (2009) have investigated the impact of ignoring APL in the frequency domain of station coordinates time series. Here, we use the model developed by Petrov and Boy (2004) — a model widely used within the VLBI-community (VLBI: Very Long Baseline Interferometry).

The Center for Orbit Determination in Europe (CODE) is interested to improve the quality of the GNSS data processing. For that reason the impact of applying APL corrections ${ }^{1}$ on relevant parameters is evaluated in this paper. Because CODE acts as one of the global analysis centers (AC) of the IGS, some additional requirements need to be considered to cover also the operational aspects of this service:

- A consistent set of corrections is necessary for the reprocessing activities at least back to 1994 .

- The final products are generated with a latency of only three days. Consequently the APL corrections are (reliably) needed with the same latency at least.

- If the ACs of the IGS start using APL corrections to generate their products, the users of the IGS products will ask for such corrections (e.g., to keep the consistency of a Precise Point Positioning, PPP, Zumberge et al. 1997), as well.

- Because of the substantial number of GNSS sites (in particular outside the IGS) an open access of the APL model in grids with a sufficient spatial resolution is required. Providing corrections as time series for individual stations (as it is done, e.g., for VLBI) is not feasible.

These requirements were considered, e.g., when selecting the APL model and its representation.

The set of GNSS stations included in the CODE reprocessing effort (details are provided in Sect. 2.1) is used here

\footnotetext{
${ }^{1}$ For GNSS measurements only corrections for the vertical and horizontal site displacement as provided by an APL model are relevant. Therefore, we use the term APL "corrections" subsequently without stating each time that these are the displacement corrections.
}

for validating APL corrections, to compare different methods to consider the APL effect in the GNSS data analysis, and to assess the impact of APL on some selected parameters of the GNSS data processing. The density and the global distribution of the GNSS sites in combination with the nearly continuously available observations make this material an ideal data set to study APL. The APL model by Petrov and Boy (2004) is described in Sect. 2.2.

The weekly GNSS solutions are compared to weekly mean values emerging from the APL model in Sect. 3.1. In a second step the corrections from the APL model are directly applied to the individual observations in the parameter estimation process. To assess the quality of the corrections, scaling factors for the APL corrections from the model are estimated as additional parameters. The results are discussed in Sect. 3.2.

As an alternative to introducing a geophysically derived APL model, one may estimate regression factors between the local pressure and the station displacement; this approach is discussed in Sect. 4. The study is concluded in Sect. 5 by comparing the repeatabilities of the coordinate time series based on the different strategies of applying the APL corrections. Because these results are derived from processing a global and dense GNSS network, the impact of correcting or not correcting for the APL effect on global parameters (datum definition and GNSS satellite orbits) is discussed in Sect. 6.

\section{Data sets}

\subsection{GNSS solution}

CODE is a joint venture of the Astronomical Institute of the University of Bern (AIUB, Bern, Switzerland), the Swiss Federal Office of Topography (swisstopo, Wabern, Switzerland), the Federal Agency for Cartography and Geodesy (BKG, Frankfurt am Main, Germany), and the Institut für Astronomische und Physikalische Geodäsie of the Technische Universität München (IAPG/TUM, Munich, Germany). All operational computations related to the IGS are performed at the AIUB using the latest development version of the Bernese GPS Software (Dach et al. 2007).

CODE participated in the first reprocessing effort of the IGS. The corresponding computations were performed at IAPG/TUM between summer 2008 and spring 2009 (Steigenberger et al. 2009b). The cleaned observation files, including the resolved integer carrier phase ambiguities, were used to generate a new set of daily solutions by making use of the latest CODE processing models and strategies (Schaer et al. 2008): satellite orbit parameters ${ }^{2}$ only for GPS, Earth

\footnotetext{
${ }^{2}$ Six initial osculating elements and nine empirical parameters are set up for each satellite arc. The empirical parameters consist of three
} 
rotation parameters (with daily time resolution), station coordinates, and troposphere parameters are set up and estimated from the data. Because it is the usual practice in the IGS (but also for the other space-geodetic services), we derive weekly solutions by stacking the daily normal equation systems and by solving the resulting normal equation system. The procedure results in 783 weekly normal equation systems covering the time interval between GPS weeks 0729 (January 1994) and 1511 (December 2008).

The weekly solutions are subsequently stacked to generate a cumulative solution. Outliers and discontinuities are automatically detected in this solution using the program FODITS (acronym for "find outliers and discontinuities in time series", Ostini et al. 2008), a new component of the Bernese GPS Software. Linear station motion is taken into account by this program. The datum definition is realized by minimum constraints strategy by applying no-networkrotation conditions with respect to the IGS05 (an IGS-specific realization of ITRF2005, Altamimi et al. 2007) on a set of reference stations verified by inspecting the residuals of a Helmert transformation. For all solutions in this study the same outliers and discontinuities were identified and removed, and the same reference sites were used.

According to Steigenberger et al. (2009a) parts of the APL deformation may be absorbed by the troposphere modeling in the GNSS data processing. In contrast to the operational processing at CODE we use, therefore, the Vienna Mapping Function 1 (VMF1, Böhm et al. 2006b) and a priori hydrostatic zenith path delays derived from the European Center for Medium-Range Weather Forecasts (ECMWF), provided as a component of the VMF1. These coefficients are interpolated from the grid files $\left(2.0^{\circ} \times 2.5^{\circ}\right.$ every $\left.6 \mathrm{~h}\right)$ and corrected for the actual station height according to Kouba (2008).

\subsection{Time series of atmospheric pressure loading corrections}

The variation of the mass distribution in the atmosphere causes changes in the loading deformation of the Earth surface, where the mass distribution can be expressed by the distribution of the surface pressure $p(\varphi, \lambda, t)$ as, e.g., provided by global weather models. The permanent deformation caused by the long-term mean pressure field $\bar{p}(\varphi, \lambda)$ is included in the station coordinates of the reference frame. For that reasons only the deviation $\Delta p(\varphi, \lambda, t)=p(\varphi, \lambda, t)-$

\footnotetext{
Footnote 2 continued

constant and six once-per-revolution parameters in the Sun-oriented coordinate system according to the orbit model described in Beutler et al. (1994). Four of the periodic parameters (the $D$-components pointing from the satellite to the Sun and the $Y$-components going along the solar panel axis of the satellite) are heavily constrained to zero. Empirical velocity changes (so-called pseudo-stochastic pulses) are set up in three orthogonal directions, constrained, and solved for at 12-h intervals.
}

$\bar{p}(\varphi, \lambda)$ needs to be considered for the loading computation. The relation between the pressure variations $\Delta p(\varphi, \lambda, t)$ and the vertical deformation $\zeta_{u p}(\varphi, \lambda, t)$ is given by the so-called Green's Function $\mathfrak{G}_{r}(\cos \beta)$ characterizing the deformability of the Earth (load love numbers $h_{n}^{\prime}$ ) and considering the angular distance $\beta$ of the mass from the point of deformation by a development of Legendre polynomials of $\mathfrak{P}_{\mathfrak{n}}(\cos \beta)$ :

$$
\begin{aligned}
\zeta_{\text {up }}(\varphi, \lambda, t) & =\int_{A} \frac{\Delta p\left(\varphi^{\prime}, \lambda^{\prime}, t\right)}{g} \cdot \mathfrak{G}_{r}(\cos \beta) \mathrm{d} A \\
\mathfrak{G}_{r}(\cos \beta) & =\frac{G \cdot R}{g} \sum_{n=1}^{\infty} h_{n}^{\prime} \cdot \mathfrak{P}_{\mathfrak{n}}(\cos \beta)
\end{aligned}
$$

where $G$ is the constant of gravitation, $R$ the mean radius of the Earth, and $g$ the mean surface gravity according to the used Earth model. $\left(\varphi^{\prime}, \lambda^{\prime}\right)$ denotes the location of the surface element $\mathrm{d} A$. Analog loading functions exist for the horizontal components (see, e.g., Farrell 1972).

The time series of displacements caused by the APL provided by the Service of the Atmospheric Pressure Loading run by NASA GSFC (http://gemini.gsfc.nasa.gov/aplo, Petrov and Boy 2004) were extracted from a global grid representation with a spatial resolution of $2.5^{\circ} \times 2.5^{\circ}$ and a time resolution of $6 \mathrm{~h}$. They are provided in the center of mass (CM) frame (Blewitt 2003). The corresponding $S_{1}$ and $S_{2}$ constituents of the displacements caused by the APL computed by Petrov and Boy $(2004)^{3}$ have been applied at the observation level.

By introducing geophysical models into the GNSS data processing their impact on the reference frame realization needs to be investigated. This seems to be in particular necessary, because the time interval used for this solution differs from the interval used by Petrov and Boy (2004) to compute the reference pressure field $\bar{p}(\varphi, \lambda)$. The time-averaged corrections from the APL model are computed for all 240 stations, which are included into the GNSS processing over the entire interval. These mean values are below $0.1 \mathrm{~mm}$ indicating that the reference pressure field in the background of the APL model is sufficiently accurate to avoid a negative impact on the reference frame realization.

The next step consists of evaluating the order of magnitude of the APL corrections for the stations of the network. The RMS of the APL corrections for the stations of the GNSS solution over the 15-year interval is shown in Fig. 1. The figure confirms that the vertical deformations (Fig. 1a) are small for stations close to the coast line and larger for in-land stations. On the other hand, the horizontal

\footnotetext{
3 According to Petrov and Boy (2004) four parameters have been estimated for each grid point from the surface pressure fields over the time period from 1980 to 2002: a mean pressure to realize a reference pressure field $\bar{p}(\varphi, \lambda)$, sine and cosine amplitude of the $\mathrm{S}_{1}$, and cosine for $S_{2}$ constituent to extract the atmospheric tidal signal.
} 
Fig. 1 Standard deviation of the APL corrections from the Petrov and Boy (2004) model over 15 years (January 1994 to December 2008) for the 240 stations included in the GNSS solution. a Vertical component. b Horizontal component
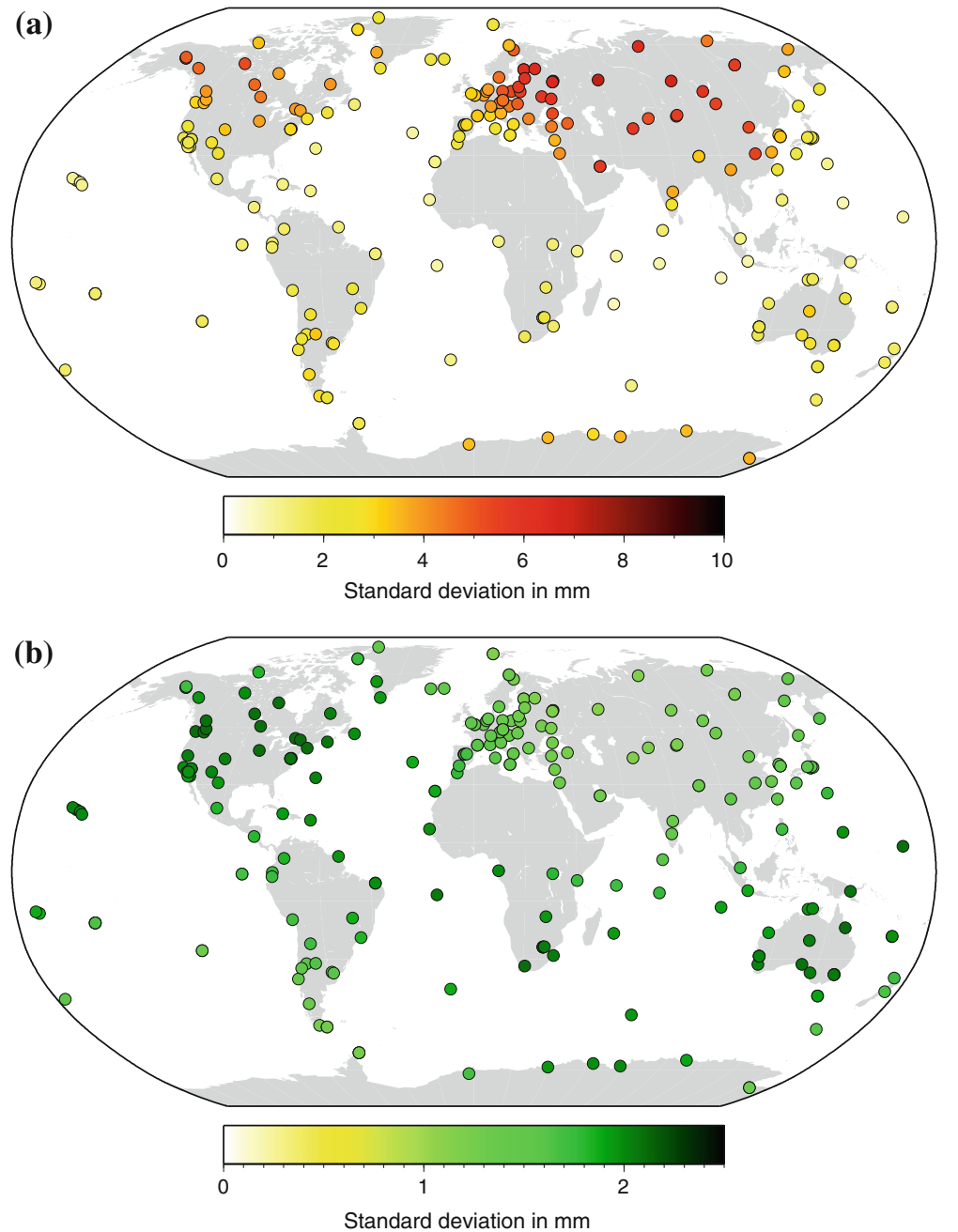

deformations $\left(\sqrt{\mathrm{d} n_{i}^{2}+\mathrm{d} e_{i}^{2}}\right.$, Fig. 1b) are a magnitude smaller than the vertical deformations. The biggest horizontal deformations can in general be found for stations located at the coasts of the big continents. This behavior is a consequence of the inverse barometer hypothesis used in the APL model by Petrov and Boy (2004). As expected, pressure variations are larger at higher latitudes than at the equator.

Figure 1a also shows that the size of the vertical corrections is substantially different for the Northern and Southern hemispheres, a consequence of the different ratio between continental and oceanic areas in the two hemispheres; but also the magnitude of the horizontal deformations in Fig. $1 \mathrm{~b}$ is not equally distributed. This fact implies that the mean vertical and horizontal corrections over the grid of the APL model can be translated into a variation of the geocenter coordinates (GCC). To assess this effect the corrections for the deformations in the North, East, and Up direction $\left(\mathrm{d} n_{i}, \mathrm{~d} e_{i}, \mathrm{~d} u_{i}\right)$ from all grid points $i$ (located at the latitude $\varphi_{i}$ and longitude $\lambda_{i}$ ) are converted into cartesian geocentric coordinates
( $\left.\mathrm{d} x_{i}, \mathrm{~d} y_{i}, \mathrm{~d} z_{i}\right)$ and summed up over all $m$ grid points for each epoch $t_{n}$ :

$$
\begin{aligned}
& \left(\begin{array}{l}
\mathrm{d} X_{i}\left(t_{n}\right) \\
\mathrm{d} Y_{i}\left(t_{n}\right) \\
\mathrm{d} Z_{i}\left(t_{n}\right)
\end{array}\right)=\Re\left(\varphi_{i}, \lambda_{i}\right) \cdot\left(\begin{array}{l}
\mathrm{d} n_{i}\left(t_{n}\right) \\
\mathrm{d} e_{i}\left(t_{n}\right) \\
\mathrm{d} u_{i}\left(t_{n}\right)
\end{array}\right) \\
& \left(\begin{array}{l}
\mathrm{d} X\left(t_{n}\right) \\
\mathrm{d} Y\left(t_{n}\right) \\
\mathrm{d} Z\left(t_{n}\right)
\end{array}\right)=\frac{1}{m} \sum_{i=1}^{m}\left(\begin{array}{l}
\mathrm{d} X_{i}\left(t_{n}\right) \\
\mathrm{d} Y_{i}\left(t_{n}\right) \\
\mathrm{d} Z_{i}\left(t_{n}\right)
\end{array}\right)
\end{aligned}
$$

with the rotation matrix

$\Re\left(\varphi_{i}, \lambda_{i}\right)=\left(\begin{array}{ccc}-\sin \left(\varphi_{i}\right) \cos \left(\lambda_{i}\right) & -\sin \left(\lambda_{i}\right) & \cos \left(\varphi_{i}\right) \cos \left(\lambda_{i}\right) \\ -\sin \left(\varphi_{i}\right) \sin \left(\lambda_{i}\right) & \cos \left(\lambda_{i}\right) & \cos \left(\varphi_{i}\right) \sin \left(\lambda_{i}\right) \\ \cos \left(\varphi_{i}\right) & 0 & \sin \left(\varphi_{i}\right)\end{array}\right)$

The result — the times series of $\mathrm{d} X(t), \mathrm{d} Y(t), \mathrm{d} Z(t)$-is provided in Fig. 2, which shows a variation of almost $5 \mathrm{~mm}$ in magnitude in the $Z$-component. The $Y$-component shows 


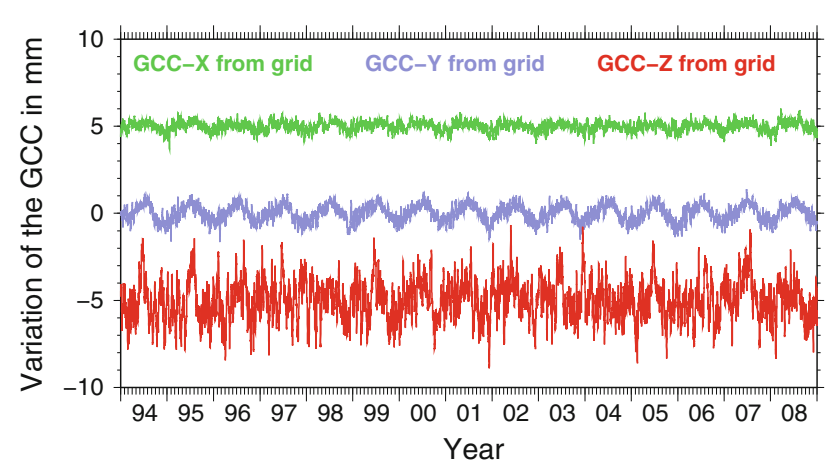

Fig. 2 APL corrections from the $2.5^{\circ} \times 2.5^{\circ}$ grid, translated into variations of the GCC. Individual components were shifted by $5 \mathrm{~mm}$

a clear annual variation as well, but only with an amplitude of about $2 \mathrm{~mm}$. The effect for the $Y$-component is in turn larger in size than the effect for the $X$-component (which is below $1 \mathrm{~mm}$ ).

As most of the sites in our solution (as most of the spacegeodetic tracking sites) are located on the Northern hemisphere, the variations of the GCC induced by the APL effect are amplified when ignoring APL in the data analysis. It is the basic assumption of the GNSS data processing that the center of mass as it is realized by the satellite orbits and the origin of the terrestrial reference frame (as realized by the coordinates of the ground stations) coincide. Otherwise, the obtained satellite orbits may be effected. This aspect will be discussed in Sect. 6.2.

\section{Atmospheric pressure loading and coordinate time series}

\subsection{Comparisons based on weekly GNSS solutions}

A simple way of validating the APL model consists of estimating correlation factors given by the slope of a linear regression between the variation of the station heights in the weekly GNSS solutions and in the weekly mean vertical APL corrections derived from the model. The results are provided in Fig. 3. The colors indicate the size of the APL effect as provided by the RMS of the corrections (see Fig. 1a). Only stations with large APL corrections (RMS $\geq 4 \mathrm{~mm}$ ) and with at least 300 weekly solutions have been included.

Figure 3 shows that most of the correlation factors are between 0.5 and 0.9 and that they are on the average about 0.8 (instead of 1.0 as expected for a perfect model). Note that a $20 \%$ deviation from 1.0 corresponds on average to a change of about $1 \mathrm{~mm}$ in the vertical station coordinate. It is therefore difficult to decide whether the APL model has deficiencies or whether the deviations have to be attributed to the GNSS analysis (e.g., receiver and satellite antenna calibration, Schmid

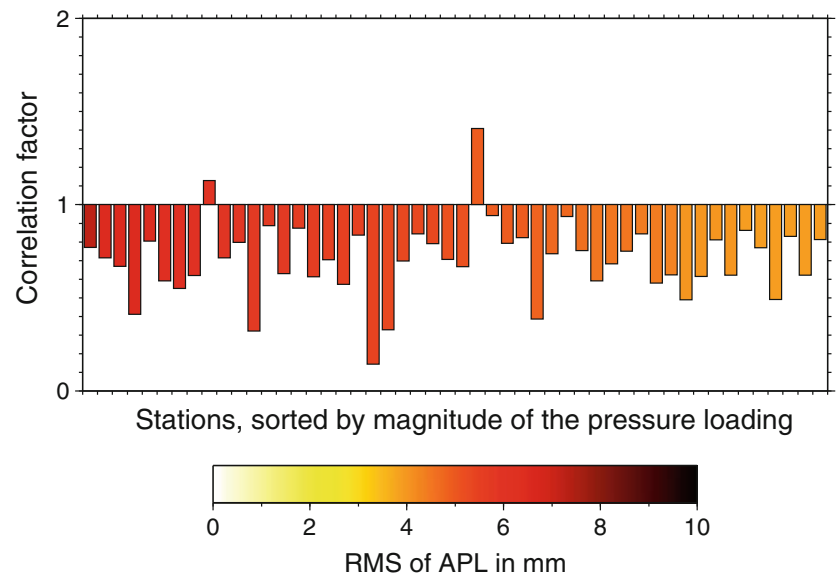

Fig. 3 Correlation factors between the time series of weekly height variations of the GNSS solution and weekly mean APL corrections from the Petrov and Boy (2004) model. The colors indicate the size of the APL effect for the stations (in units of the RMS of the corrections from the model over 15 years)

et al. 2007). It is also possible that the neglected APL corrections are distributed to other sites due to the datum definition of the weekly solutions (see Böhm et al. 2009, and Fig. 2).

One should also keep in mind that this method is based on weekly mean coordinate sets derived from GNSS, which are compared to weekly mean APL corrections. The APL correction is computed without applying epoch-specific weights. When calculating the APL corrections, it is therefore assumed that the number of GNSS observations is constant throughout the entire week. In the case of GNSS analysis based on real data this is clearly not true because of temporal data outages. In addition, the number of satellites tracked by the stations varies roughly between 8 and 12 satellites throughout a day in the current GPS constellation for midlatitude sites.

Böhm et al. (2009) state that the variation of the APL effect within $24 \mathrm{~h}$ may be significant compared with the magnitude of the effect itself. It is therefore necessary to check the impact of our simplifying assumption resulting from the weekly processing schedules of the space-geodetic services. These results are presented in Sect. 3.2. There are, however, also possible advantages of the simplified procedure as they are discussed, e.g., by Collilieux et al. (2010): The GNSS solution itself does not contain any APL model. (1) Solutions based on the same space-geodetic technique or (2) solutions based on different techniques may be corrected after data analysis and before combination in a consistent way using identical values. This aspect may be important for the rigid processing scheme applied within the IGS, because a dependency on the external generation of the APL corrections may be introduced due to different latencies of the APL and GNSS products. If, e.g., the final solution is running 3 or 4 days behind real time, the latency of the APL corrections available 
at http://gemini.gsfc.nasa.gov/aplo/ is not sufficient (APL latencies of more than one week occur occasionally). For a GNSS reprocessing effort such latencies are, of course, no issue.

\subsection{Comparisons by direct estimation of scaling factors}

The APL corrections are directly applied to the observations in the GNSS analysis in this section and scaling factors for the vertical and the horizontal components are estimated for each station. The corresponding additional parameters are stacked when combining the daily and weekly solutions to the long-term GNSS solution as described in Sect. 2.1. Note that these scaling factors are considered as constants for a station, even if a discontinuity had to be introduced in the coordinate time series.

The resulting scaling factors are provided in Fig. 4 and the colors are related to the RMS of the APL correction over the time interval of 15 years. Only sites available in at least 300 weekly solutions and with a minimum RMS in the APL of $4 \mathrm{~mm}$ are included. The ordering of the stations is the same as in Fig. 3. The deviation from the expected scaling factor of 1.0 is in general below $30 \%$. For stations with big APL effects deviations between 10 and $20 \%$ are typical. In view of the expected uncertainties of the model (15\%, Petrov and Boy 2004) and the GNSS solutions (week-to-week repeatability for the vertical component of about $1.5 \mathrm{~mm}$ for the best stations, Schaer et al. 2008) the APL model may be considered as "confirmed" by the GNSS solution.

The scaling factors displayed in Fig. 4 (estimated from the GNSS data when applying the APL corrections directly to the observations) confirm the APL model whereas this is not the case for the correlation factors in Fig. 3 (based on weekly mean station deformations and APL corrections).

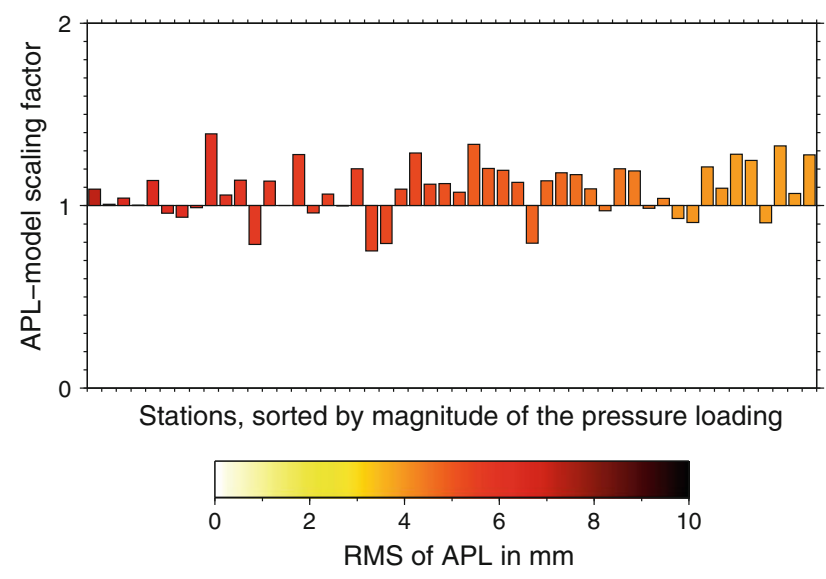

Fig. 4 Scaling factors for the APL corrections from the Petrov and Boy (2004) model computed from 15 years of GNSS processing. The colors indicate the size of the APL effect for the stations (in units of the RMS of the corrections from the model over 15 years)
This implies that directly correcting the observations for APL is preferable to applying only weekly mean APL corrections to weekly coordinate solutions.

The direct APL correction on the observation level removed the systematic offset of the correlation factors obtained when correcting only weekly mean coordinate solutions. The relative station-to-station relation seen in the two figures is rather similar for most of the stations, which implies that part of the APL effect has been absorbed by other parameters (or even the NNR-condition) when ignoring the effect in the analysis. When correcting the weekly solutions by weekly mean APL values, an "over-correction" of the effect takes place. This phenomenon is comparable to what (Böhm et al. 2009) showed: In the VLBI analysis the neglected APL corrections are distributed to the other stations in the network. Even if our GNSS analysis includes many more stations with a better global distribution, about two-thirds of the stations are located in the Northern hemisphere-and roughly $20 \%$ of all included sites are in Europe.

Among the stations contributing to Fig. 4 there are two receivers, both located at Kirkkonummi, Finland, namely METS and METZ. ${ }^{4}$ In the global network solution both sites have been treated as independent (the observations are used with the ionosphere-free linear combination with individual troposphere parameters). Nevertheless, "the same" results concerning APL are expected because of the closeness of the two receivers. The correlation factors associated with the weekly GNSS solutions and the weekly mean APL values from the model are 0.55 and 0.59 , respectively. The values indicate that the APL corrections stemming from the model are about twice the size of the effect seen by the GNSS analysis. In contrast to that, the estimated scaling factors for the APL model as established on the observation level are 0.94 and 0.96 , respectively, for the two receivers and agree even better than the results obtained with the weekly mean APL corrections. Nevertheless, both pairs of correlation factors and scaling factors agree within the statistical expectation.

Figure 5 gives a complete overview of the resulting scaling factors. The deviations of the scaling factors from 1.0 are scaled by the RMS of the APL corrections for each of the three components from the model (see, e.g., Fig. 1a for the vertical component) to take into account the sizes of the APL effects at the individual stations. Different occupation times for the IGS stations are characterized by different sizes of the symbols-larger circles indicate a more reliable scaling factors because of the length of the time series.

\footnotetext{
4 There are more examples for groups of GNSS receivers at the same location in the processed network, but either the magnitude of the APL effect is much smaller at these locations or fewer than 300 weekly solutions are available suggesting a higher uncertainty for the obtained scaling factors.
} 
Fig. 5 Deviation of the scaling factors from 1.0 for the APL corrections emerging from the Petrov and Boy (2004) model established by the analysis of 15 years of GNSS data scaled by the size of the APL correction for the station (in units of the RMS of the corrections from the model over 15 years). a Vertical component. b North-south component. c East-west component
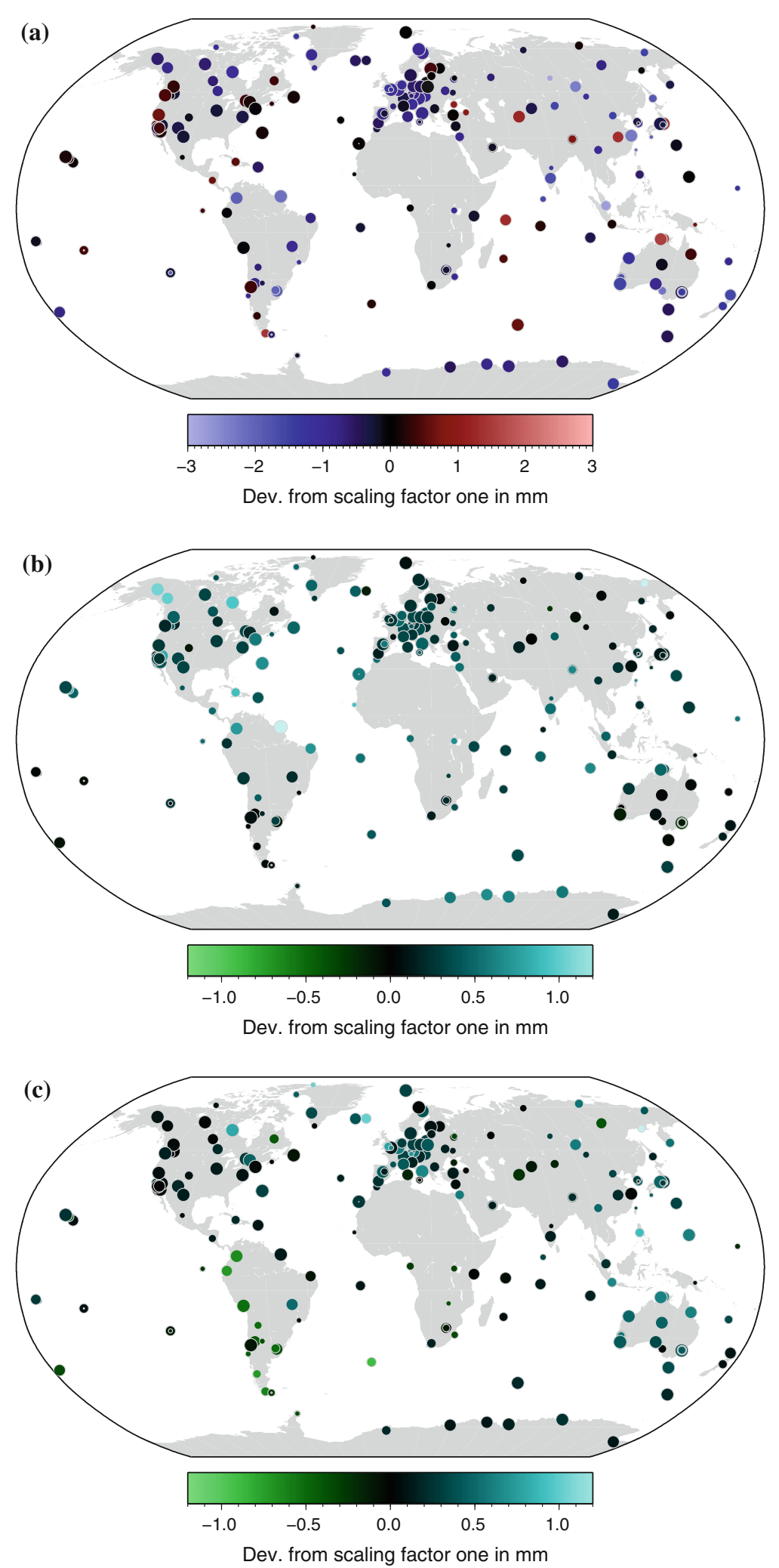

Scaling factors derived from:

015 years of data 012 years of data $\circ 9$ years of data $\quad 6$ years of data 
Figure 5a shows black or very dark dots for nearly all stations, indicating that the APL model agrees on the $1 \mathrm{~mm}$ level with the GNSS solution. On the one hand, no systematic deficiencies for the APL model values, e.g., in particular regions, could be found. On the other hand, some stations in the GNSS solution contribute only with a limited number of weeks to this validation, which is indicated by smaller circles.

Figure $5 \mathrm{~b}, \mathrm{c}$ shows the analog results for the horizontal components: a general confirmation for the APL model. Black or dark symbols are related to deviations of about $0.5 \mathrm{~mm}$ from a scaling factor of 1.0. In the North-South component bigger exceptional points (symbols with light colors, Alaska, north Canada and Kourou) are found than for the East-West component. Negative scaling factors dominate for stations in South America for the East-West component, whereas positive scaling factors are found in Australia. This fact indicates deficiencies in the reference frame realization due to the inhomogeneous station distribution in the network, in particular the extremely high concentration of stations in Europe. This effect is amplified by the simultaneous estimation of the three APL model scaling factors per station (if the APL values do not vary in time it is a one-to-one correlation).

In summary, more than $80 \%$ of the stations show a deviation of less than $0.5 \mathrm{~mm}$ for the rescaled scaling factors in the horizontal components (as displayed in Fig. 5), if only stations with a minimum data interval of 300 weeks are considered. In the case of the vertical component a deviation of less than $1 \mathrm{~mm}$ is detected for $75 \%$ of the sites.

The scaling factors are available for each station in weekly normal equations, allowing it to stack all weekly scaling factors belonging to a particular month. This results in 12 scaling factors per station referring to each month of the year. These values can be used to decide whether the APL model values agree on the same quality level for all months of the year with the corresponding estimated values. The coordinates and velocities of the cumulative solution covering 15 years with one scaling factor for the entire interval are introduced as known. This procedure prevents the estimated station coordinates and velocities to absorb parts of the annual variations of the scaling factors.

Figure 6a shows the result for the Zimmerwald site in Switzerland as a typical European station with a moderate APL effect. Figure $6 \mathrm{~b}$ shows the results for Arti, Russia, which is the station with the largest APL effect in the GNSS solution.

Large deviations from the expected scaling factor of 1.0 and also from the mean scaling factor over 15 years occur in particular for the summer months. It is very unlikely that the APL model is of lesser quality during this period than in the remaining months of the year. It is more likely that other effects of the same size as APL (e.g., ocean-induced non-tidal or continental water mass surface load) not taken
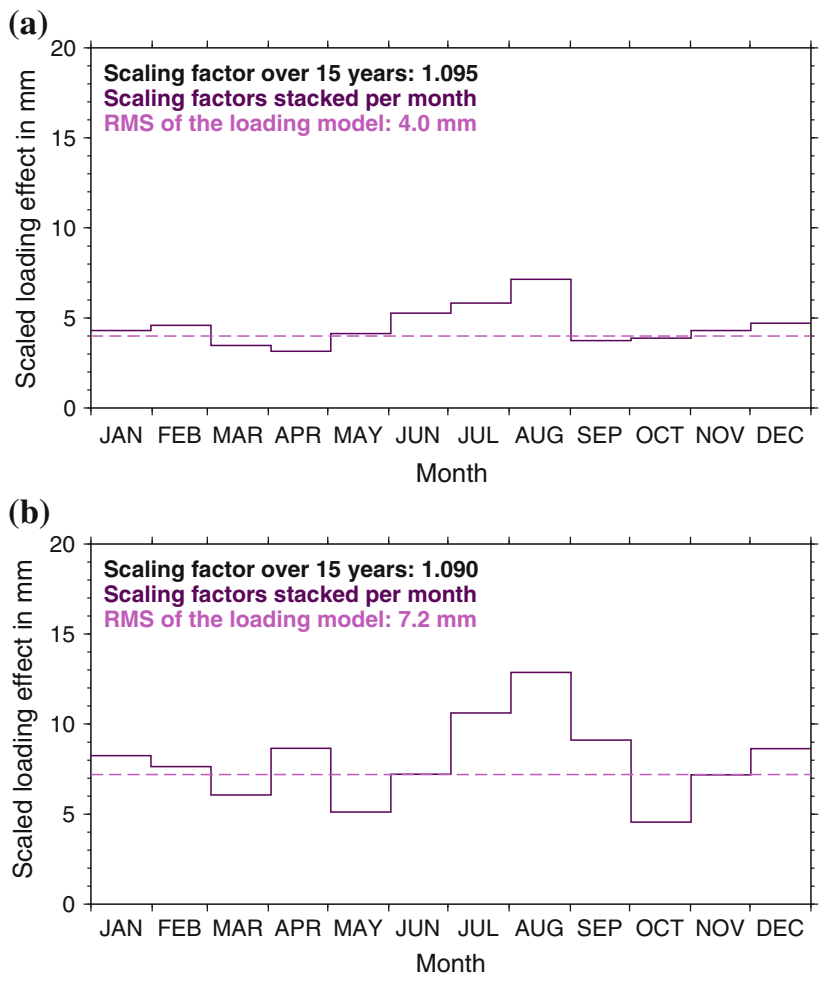

Fig. 6 Scaled loading effect per month computed by stacking the weekly scaling factors of each month over all the years to obtain 12 scaling factors per station (in units of the RMS of the APL corrections from the model over 15 years). The dashed line indicates the RMS of APL corrections-it refers to the scaling factor of 1.0. a Station Zimmerwald, Switzerland. b Station Arti, Russia

into account in the GNSS analysis, are responsible for this deviation.

\section{Atmospheric pressure loading corrections based on local pressure time series}

The APL effect on vertical station displacements is fully described in Eqs. (1) and (2). The Green's function does quickly decrease with increasing angular distance implying that the loading deformation mainly depends on the masses located close to the point for which the deformation is computed. Considering this fact, approximations have been suggested to simplify the computation of APL. Rabbel and Zschau (1985) have proposed to consider only the distribution of the atmospheric pressure in a distance of up to $2000 \mathrm{~km}$ around the point where the deformation shall be computed. But even with this approach, for each of the big numbers of GNSS tracking stations a separate loading computation is necessary before the processing of the GNSS measurements can be started. There are a number of studies (Tesmer et al. 2008; Kaniuth and Vetter 2006, and others), which solve for regression factors between the time series of local pressure and vertical station displacement to take the APL effects into 
account. Some of these studies introduce annual variations for the regression factors (e.g., Manabe et al. 1991).

It is clearly only an approximation taking only the vertical component into account and ignoring the pressure distribution around the tracking station. It has the advantage that the dependency on externally generated APL models is avoided. The local pressure values at the observing stations are a component of the troposphere delay model. They are available for the GNSS data processing, if local atmosphere values are considered for the a priori troposphere model (e.g., the hydrostatic zenith path delay as provided by Böhm et al. 2006b, which is derived from the ECMWF weather model).

By introducing time series of local pressure instead of the APL model one can also generate a comparable solution for the 240 stations of the CODE reprocessing solution. Local pressure as a function of time is extracted from the grids of VMF1 coefficients. The mean pressure for each grid point over the 15 years considered here is computed to obtain a reference pressure field. The deviation from this mean pressure field for each individual grid file is the pressure anomaly used for the estimation of regression factors in the following analysis.

\subsection{Comparison of APL corrections with local pressure data}

Before analyzing the GNSS-derived regression factors, the regression factors between the APL corrections of the Petrov and Boy (2004) model and the local pressure field are studied. A small inconsistency occurs because the APL corrections are computed from data of the NCEP (National Centers for Environmental Prediction) whereas the local pressure values are extracted from the ECMWF. According to van Dam et al. (2003) the differences between the two models are small enough not to have a significant impact on the APL modeling.

At each grid point of the APL model a regression factor relative to the local pressure is computed from the 15-year period between 1994 and 2008. The result is shown in Fig. 7a, the corresponding formal uncertainty of the regression factors in Fig. 7b. The regression factors lie between -0.3 and $-0.5 \mathrm{~mm} / \mathrm{hPa}$ for most of the continental regions. They are larger (up to $-1.0 \mathrm{~mm} / \mathrm{hPa}$ ) for central Asia. The Himalaya region, where the regression factors are even positive, is an exception. This regional anomaly currently cannot be explained, but it might be related to the realization of the reference pressure and the topography in the area. Surprisingly, the regression factors are not zero over the ocean areas close to the equator-but the formal uncertainties of the regression factors in these regions are up to ten times larger than elsewhere. These features are, however, related to the inverted barometer hypothesis realized over the oceans and need not be discussed further.
The formal uncertainty of the regression factors mainly depends on the magnitude of the variation of local pressure as a function of time. The larger the variation of local pressure values, the better the regression factors can be established. Therefore, the RMS values of local pressure of the global grid are provided in Fig. 8, which explains the regions of higher uncertainty of the regression factors in Fig. 7.

Because seasonal variations of the scaling factors for the APL model were detected in the GNSS data at the end of Sect. 3.2, the regression factors between the APL model and the local pressure were also computed on a monthly basis using 15 years of data. The 12 maps in Fig. 9 reflect the monthly mean regression factors as established over the 15year time interval. ${ }^{5}$ The largest variations occur in Africa, Central Asia, and close to the Himalaya region. At least a part of these variations may be explained by the uncertainty of the regression parameters corresponding to Figs. 7 and 8.

Real monthly variations of the regression factors cannot be explained in this experiment: it is a comparison between the local pressure and the APL model with the global pressure field as the main input parameter (apart from the coastal lines and the Green's function which are both constant in time). Possibly, the treatment of the inverse-barometer effect and the mean seasonal pressure distribution may lead to this effect.

\subsection{Regression factors from GNSS data analysis}

The grids containing local pressure are interpolated for the coordinates of the GNSS tracking stations and introduced to compute the regression factors starting from the observation level. The resulting regression factors between the time series of local pressure and vertical displacement for all 240 stations are provided in Fig. 10.

In the regions with big APL effects the regression factors are in the expected range between -0.3 and $-0.6 \mathrm{~mm} / \mathrm{hPa}$. In general these values agree well with results of studies using the same method. Our regression factors are usually in good agreement with the map in Fig. 7a. The size of the circles indicates the different occupation intervals for the IGS stations.

If the regression factors for each particular station and month of the year are considered as common parameters in the weekly normal equation systems of the 15 -year time period one obtains 12 parameters per station characterizing the mean annual variation of the station-specific factors. The values for Zimmerwald and Arti are provided in Fig. 11.

\footnotetext{
5 The mean pressure field used as basis for the local pressure values has been computed as arithmetic mean over the full interval of 15 years for each grid point. Seasonal variations are not considered. For that reason for each monthly map not only a slope (regression factor) but also an offset has been computed.
} 
Fig. 7 Regression factors between the time series of local pressure and APL model from Petrov and Boy (2004) (top) over 15 years with their formal uncertainty (bottom).

a Regression factors. b Formal uncertainties
Fig. 8 Standard deviation of the local pressure over 15 years (January 1994 to December 2008)
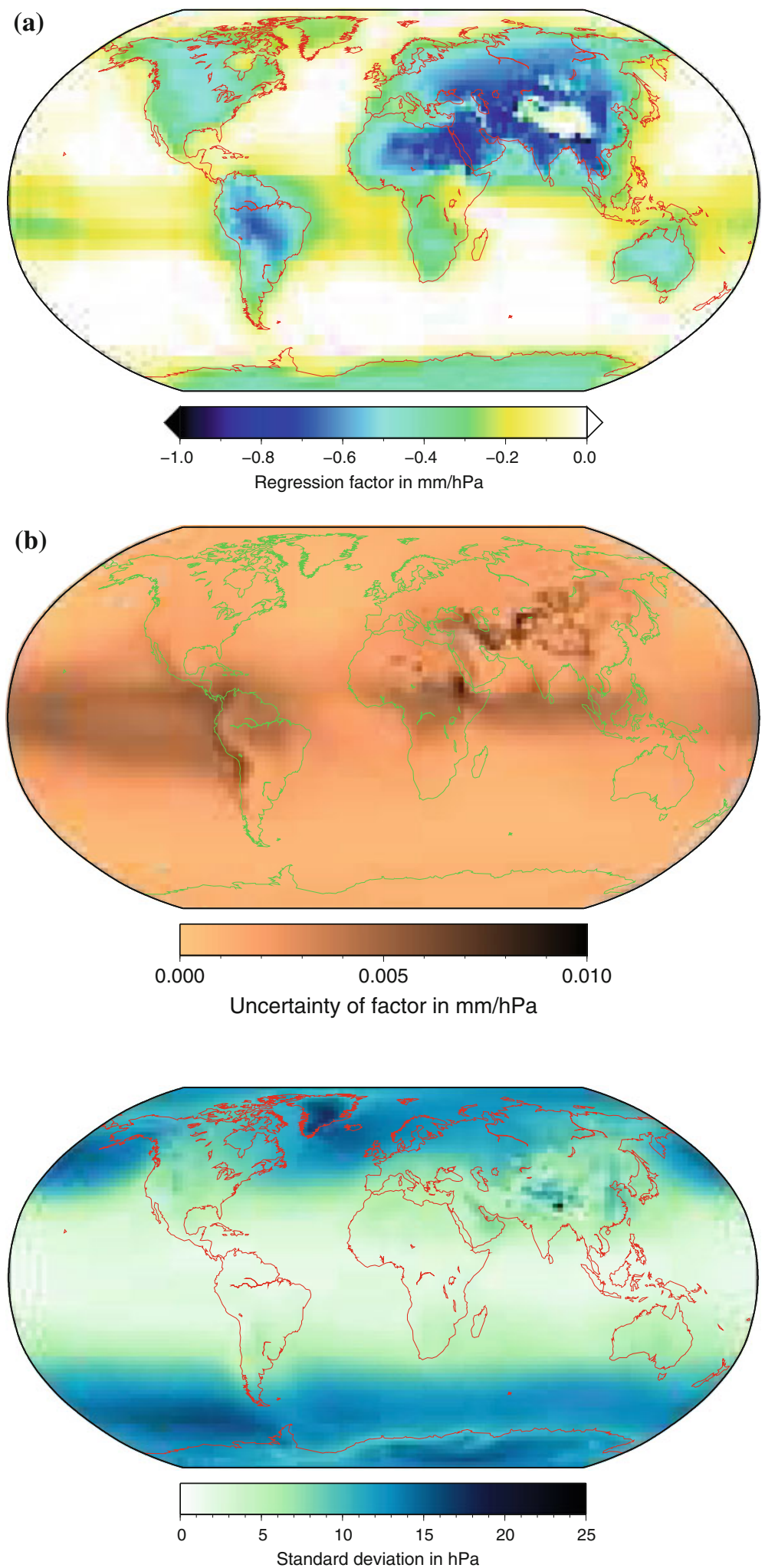

The dashed line shows the monthly regression factors between the local pressure and the APL model. The latter ones are solely based on geophysical models. They are much smoother than the results stemming from the GNSS data.
Therefore, these variations are related to other effects than APL (as already suggested in Sect. 3).

In Fig. 10 there are a few coastal stations with high regression factors of almost $-1 \mathrm{~mm} / \mathrm{hPa}$. These sites are located 

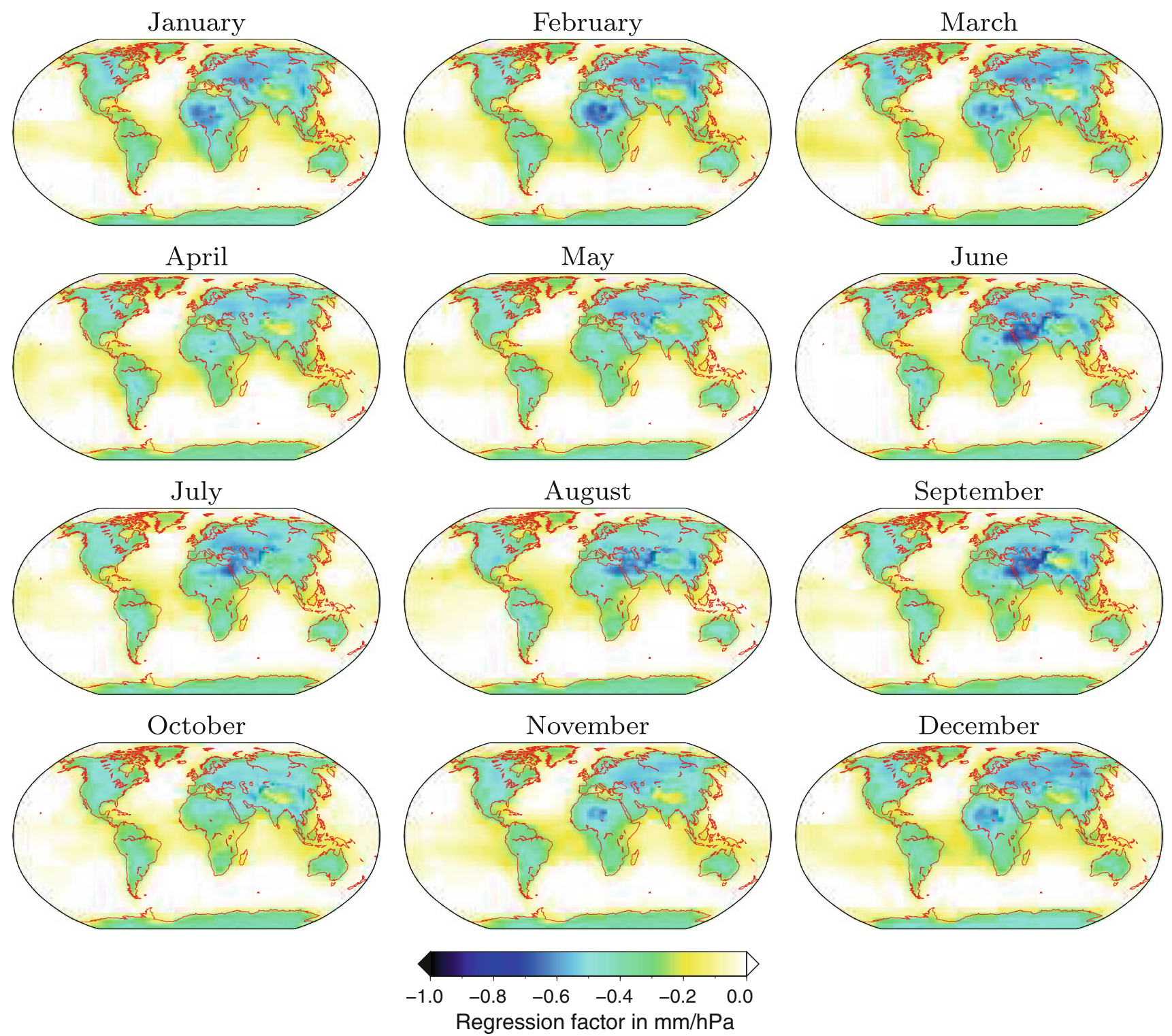

close to the equator. The APL effect, as emerging from the model, is very small, see Fig. 1. The RMS values are for Kourou (French Guyana, IGS-station ID: KOUR) $1.3 \mathrm{~mm}$, for Bogota (Colombia, BOGT) $1.5 \mathrm{~mm}$, and for La Misere (Seychelles, SEY1) $0.9 \mathrm{~mm}$. The variation of the weekly station positions is, on the other hand, rather high (but can be mostly explained by almost annual signals). The RMS of the weekly station heights for Kourou is $10.1 \mathrm{~mm}$, for Bogota $10.6 \mathrm{~mm}$, for La Misere $10.3 \mathrm{~mm}$.

Figure 8 also shows very small variations of the local pressure for these sites (RMS value for Kourou is $1.7 \mathrm{hPa}$, for Bogota $1.5 \mathrm{hPa}$, for La Misere $1.9 \mathrm{hPa}$ ). The estimation of regression factors is therefore not very reliable for these sites-the formal errors are five to ten times larger than for other sites. The estimated coefficients seem to reflect, as a matter of fact, other unmodeled effects (e.g., due to ocean non-tidal loading effect or site displacements due to continental water mass surface load, which is, e.g., very pronounced in the Amazon area, see van Dam et al. 2001, or even introduced by the GNSS data acquisition or processing). This conclusion is supported by a pronounced variation of the monthly regression factors:

Kourou minimum in June: $\quad-2.3 \mathrm{~mm} / \mathrm{hPa}$ maximum in April: $\quad+0.7 \mathrm{~mm} / \mathrm{hPa}$

Bogota minimum in July: $\quad-4.6 \mathrm{~mm} / \mathrm{hPa}$ maximum in January: $\quad+0.8 \mathrm{~mm} / \mathrm{hPa}$

La Misere minimum in December: $-3.6 \mathrm{~mm} / \mathrm{hPa}$ maximum in March: $\quad+0.6 \mathrm{~mm} / \mathrm{hPa}$ 
Fig. 10 Regression factors between the time series of local pressure and vertical site displacement from 15 years of data

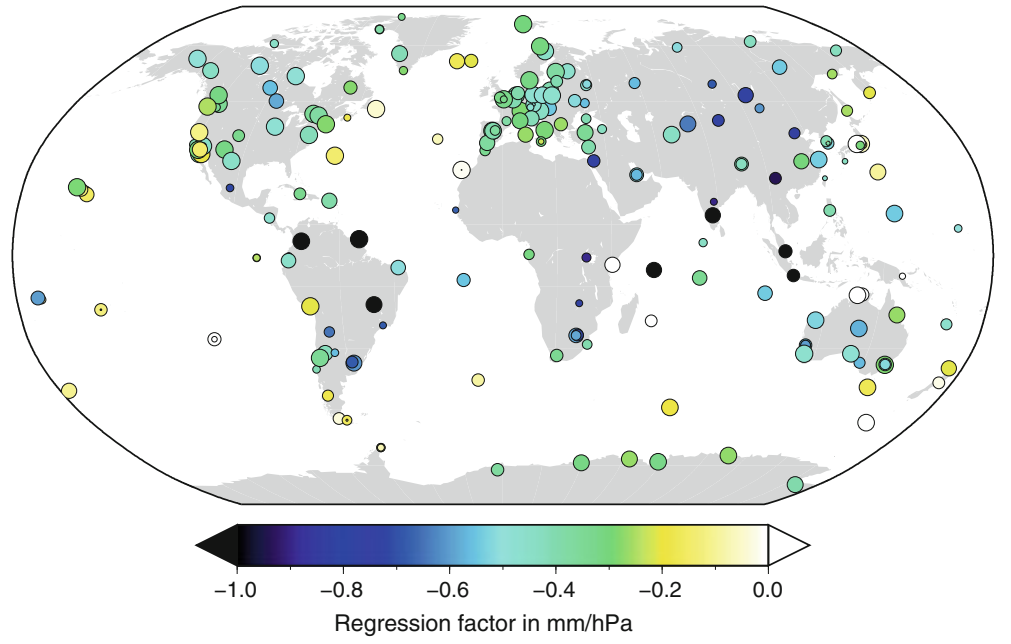

Regression factors derived from:

$\bigcirc 15$ years of data $\quad \circ 12$ years of data $\quad \circ 9$ years of data $\quad 6$ years of data (a)

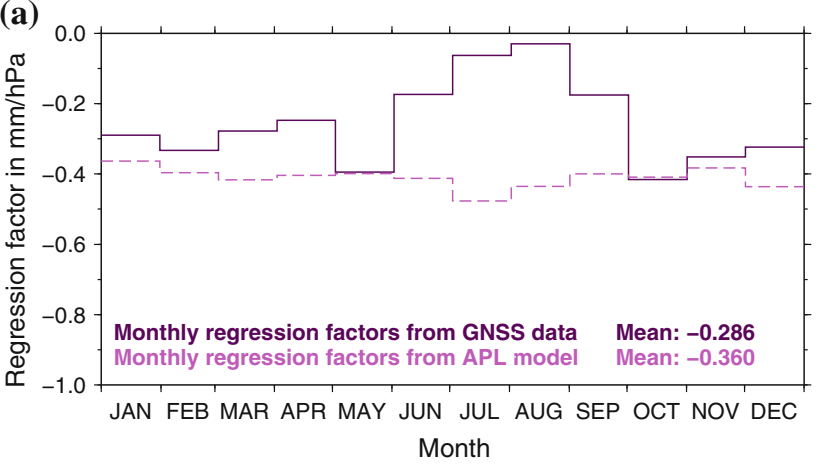

(b)

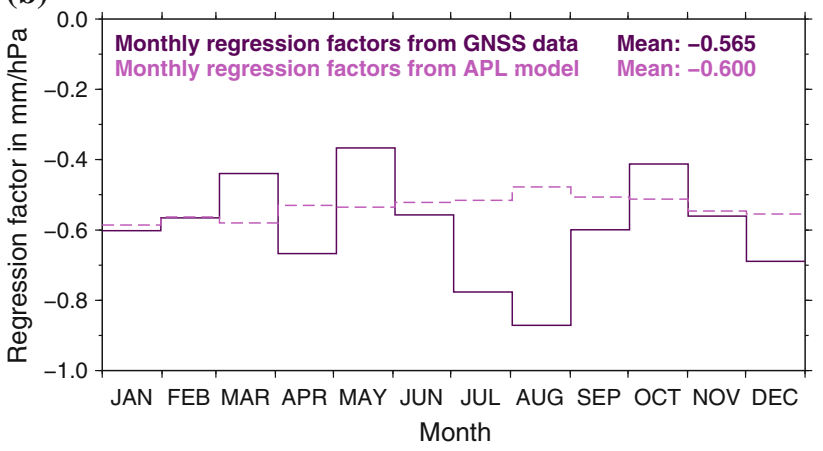

Fig. 11 Regression factors between local pressure and vertical station displacement for each month of the year(s) computed by stacking the weekly regression factors of each month to obtain 12 regression factors per station. a Station Zimmerwald, Switzerland. b Station Arti, Russia

It should also be noted that these three stations do not belong to the best performing stations in the IGS network. This increases of course the uncertainty the GNSS solution. Nevertheless, the stacking of the weekly solutions for the individual months over several years helps to smooth out this influence.

\section{Effects seen in the repeatability of station coordinates}

The repeatability (RMS of the residuals) of the weekly coordinate solutions is a good indicator to assess the benefit of a model for GNSS analysis. Without considering the APL effect it is between 4 and $6 \mathrm{~mm}$ for most of the sites-but it may reach or slightly exceed $10 \mathrm{~mm}$ for some of the stations. With a few exceptions an improvement of the repeatability of the weekly station coordinate time series between 1 and $2 \mathrm{~mm}$ can be found by taking APL into account. These are small numbers, but with respect to the repeatability of the original series without taking the APL effect into account we end up with an improvement of up to $20 \%$.

Figure 12 shows the change of the repeatability for the station heights with the largest variation in the APL considering the APL effect by the methods discussed above:

- blue: Weekly station coordinate time series are computed without considering APL but corrected by the weekly mean values from the APL model.

- red: Corrections from the APL model are directly applied to the observations during the data processing.

- violet: APL-corrections are again applied on the observation level but the estimated scaling factor for the APL model for the station is used to rescale the APL model.

- green: APL is considered by estimating regression factors between the station height and the local pressure for each station.

In agreement to the findings in Tregoning and van Dam (2005) the improvement of the repeatability is better if the corrections are applied directly to the observations during 


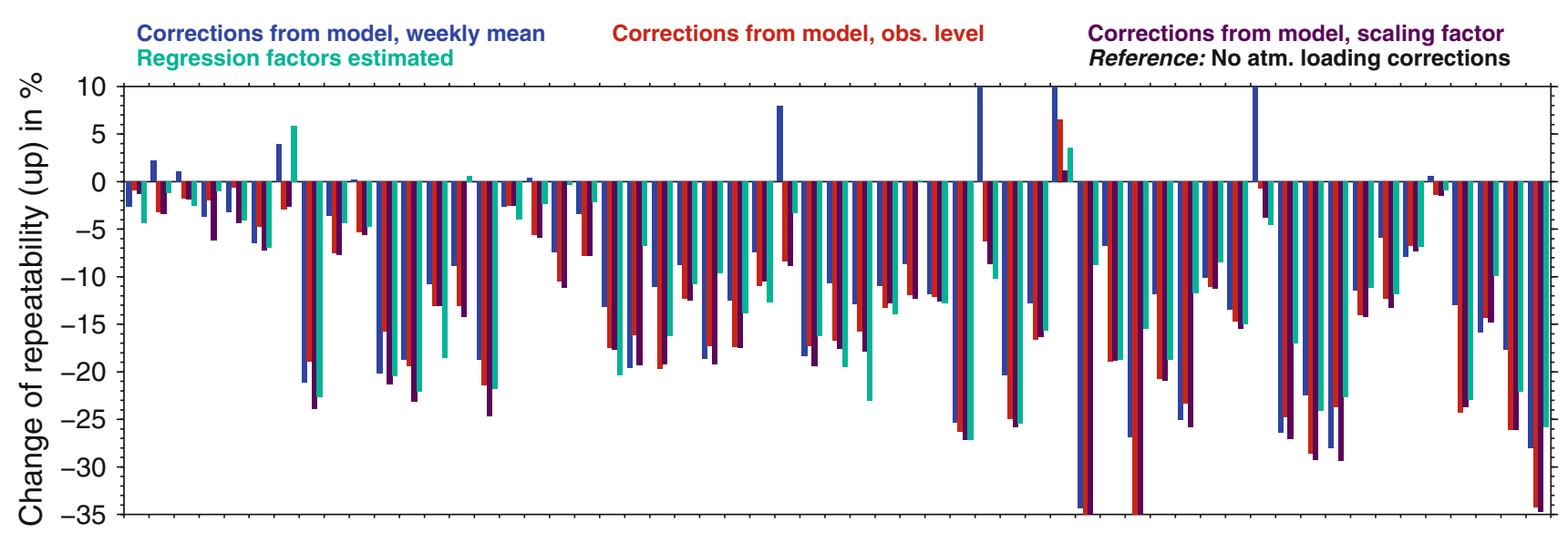

Stations, sorted by magnitude of the pressure loading

Fig. 12 Improvement/degradation of the repeatability of the station heights (RMS of residuals) as derived from the weekly solutions over the 15 years considering the APL effect in different ways with respect to the solution ignoring APL. Stations with a RMS of larger than $4 \mathrm{~mm}$ in the APL corrections in the Petrov and Boy (2004) model over 15 years are given in descending order of the size of the APL-induced effect the data processing (red bars) than obtained when correcting the weekly coordinate solutions for mean APL values (blue bars). In some cases the original APL corrections are worse (red bars) than the rescaled APL corrections (violet bars).

For many stations the direct application of the APL corrections is more effective than the use of regression factors between time series of the local pressure and the vertical site displacement (green bars). This result is related to the fact that not only the pressure at the location of the station but also in the surrounding area has an important impact on the APL for a site. Even if the horizontal component of APL is completely ignored by this strategy, there is a marginal improvement on the station repeatability also for North and East component (up to $0.1 \mathrm{~mm}$ ).

In summary over all about 50 stations in Fig. 12 we find only one case where an ignored APL effect gives the best result (all bars in the positive range) and only three examples, where the repeatability favors correcting the APL by mean corrections on the estimated weekly coordinates (blue bars). For 11 stations the best repeatability is achieved by solving for regression factors instead of applying the corrections from the APL model (green bars). For most of the stations the use of the APL model corrections on the observation level thus provides the best repeatability (red or violet bars). In 36 cases (about two thirds of the stations) both versions of the APL corrections on the observation level (the original APL model and the rescaled APL model) are slightly better than applying the APL via regression factors (the green above the red and violet bars).

That the rescaled APL corrections (violet bars) are significantly better for some of the stations than the direct APL corrections (red bars) - in particular if only the violet bars are below the green bars (eleven stations)—should not let us conclude that it is better to apply the rescaled APL corrections for a routine data processing, in particular for deriving a reference frame. Because the scaling factors were estimated station-by-station their influence on the global parameters is unclear. These results should motivate a more detailed investigation of the stations-specific time series, including

- the review whether the GNSS data processing has any deficiencies, e.g., in handling multipath effects,

- the check whether several stations in the same region are affected similarly to review the quality of the input data for the APL computation, or

- the check whether the APL effect is correlated with other geophysically induced crustal deformations (e.g., due to ocean non-tidal loading).

The repeatability of the horizontal components in the weekly solutions without applying any APL correction is on the order of $2-3 \mathrm{~mm}$ (up to $5 \mathrm{~mm}$ for a small number of stations). The repeatability is only changed by a tenth of a millimeter or even less when correcting for APL displacements. This implies that the APL corrections have no significant impact on the repeatability. Nevertheless, it is worth mentioning that applying APL corrections on the basis of weekly mean values degrade the repeatability for nearly all stations, whereas it is slightly improved for all other methods to consider APL. There are only 15 out of 50 stations where the repeatability has been improved by at least $5 \%$. On the other hand, the repeatability for the horizontal component has not been degraded if the corrections from the APL model have been applied on the observation level, what can also be interpreted as a confirmation of the APL model. 


\section{Influence of APL on global parameters}

\subsection{Influence on geodetic datum definition}

The two series of weekly coordinates with APL corrections from the Petrov and Boy (2004) model and time series without APL corrections may be compared by a seven-parameter Helmert transformation. The translations should be comparable to the variations of the GCC derived from the APL corrections. Both time series are provided in Fig. 13. For the purpose of comparison the variations of the GCC are computed from the APL model corrections at the locations of the GNSS stations. This reference time series therefore is noisier than the one computed using all grid points (see Fig. 2).

The two curves are in good agreement even though the GNSS-derived variations of the GCC show larger variations in the $Y$ - and $Z$-components than the time series derived from the APL model. This finding implies that the APL effect is not absorbed by other parameters than station coordinates. The full APL effect remains in the station coordinate parameters or can be absorbed by the datum parameters when not correcting the data for APL. According to Steigenberger et al. (2009a), the use of ECMWF-derived a priori troposphere delays together with the VMF1 prevented a compensation of the APL effect by the troposphere modeling in the GNSS analysis as it has been observed by the use of the Global Pressure and Temperature model (GPT, Böhm et al. 2007) together with the Global Mapping Function (GMF, Böhm et al. 2006a).

\subsection{Influence on GNSS-satellite orbits}

The variations in the GCC discussed in Sect. 6.1 can be compensated by additional no-network-translation conditions for the minimum constraints datum definition to generate fully

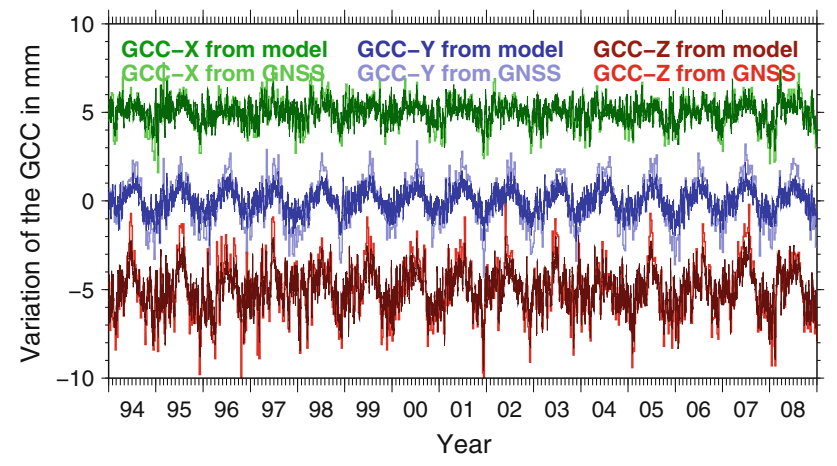

Fig. 13 Variations of the GCC from stacking the APL corrections from the APL model at the locations of the GNSS-tracking stations (light curves) and the difference of the translations in the datum definition of the weekly solutions between a solution corrected for APL and another without correcting APL (dark curves). Individual components are shifted by $5 \mathrm{~mm}$ consistent realizations of the terrestrial reference frame for series of daily solutions, one without and the other one with APL corrections on observation level. If the GNSS satellite orbits of these two solutions are given by discrete positions of the satellites every $15 \mathrm{~min}$ in the Earth-fixed frame, they are fully consistent with the coordinate sets of the station coordinates. The two sets of satellite positions computed with and without APL corrections are consequently forced to be in the same reference frame.

The orbits of two consecutive days, $i-1$ and $i$, are expected to have identical positions for each satellite for the midnight epoch $t_{i}: \mathbf{r}_{\mathbf{i}-\mathbf{1}}\left(t_{i}\right)=\mathbf{r}_{\mathbf{i}}\left(t_{i}\right)$. The resulting discontinuities $\left|\mathbf{r}_{\mathbf{i}}\left(t_{i}\right)-\mathbf{r}_{\mathbf{i}-\mathbf{1}}\left(t_{i}\right)\right|$ may serve as a quality indicator. The sum of discontinuities over all days $\sum\left|\mathbf{r}_{\mathbf{i}}\left(t_{i}\right)-\mathbf{r}_{\mathbf{i}-\mathbf{1}}\left(t_{i}\right)\right|$ do not show any significant effect whether the APL corrections are applied or not.

Applying the APL corrections on the observation level or ignoring APL results in differences in the satellite positions of 3 to $4 \mathrm{~mm}$ RMS on average. At least a part of these differences may be explained by daily computed translation between the sets of satellite positions ( $1 \mathrm{~mm}$ in $Y$ - and $4 \mathrm{~mm}$ in the $Z$-component). This seems to be in contradiction to the fully consistent station coordinate sets from both solutions which are both computed with the same datum realization and show no systematic differences in terms of transformation parameters (even no translations because of the no-network-translation conditions).

Figure 14a shows the translation parameters between the two sets of GNSS satellite positions (dark curves). They are compared to the variation of the GCC (light curves) that have already been introduced in Fig. 2. At least for the interval after the year 2000 the $X$ - and $Y$-translation components are in phase but smaller than the APL corrections translated to the GCC. This is supported by the spectra of the time series provided in Fig. 15a.

Dach et al. (2008) have shown that the realization of the origin depends on the parametrization and on the constraining of the once-per-revolution orbit parameters for the GNSS data processing. Assuming that this mechanism works in both directions, the APL-induced variation of the GCC may translate into the satellite orbits if the once-per-revolution terms are not sufficiently constrained.

This can easily be verified by inspecting another pair of solution series where the once-per-revolution terms of the orbit model are estimated without applying any constraints. The effect of the APL corrections on this solution with the alternative orbit determination scheme is shown in Figs. 14b and $15 \mathrm{~b}$, respectively. Nearly the full variation of the GCC introduced by the APL effect is absorbed by these orbit parameters. The influence of the alternative results of these terms is reflected by the translations computed between the two sets of satellite positions, with and without APL corrections at the observations during the data processing. 


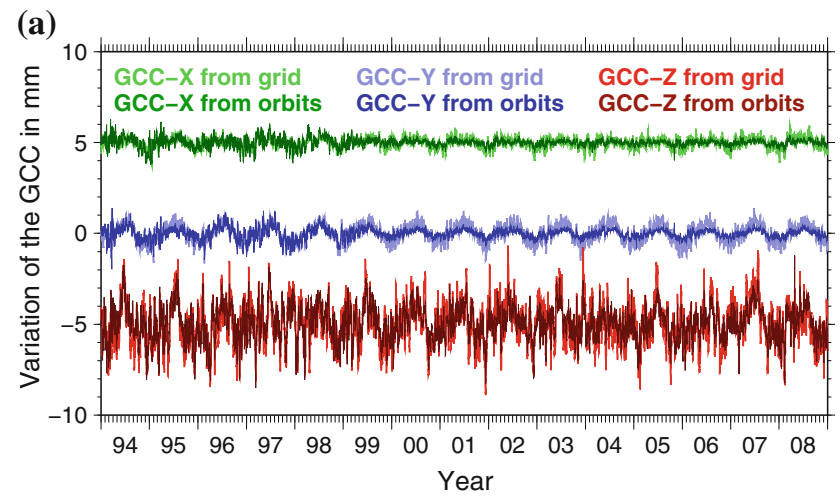

Fig. 14 Translations between the satellite positions computed with and without correcting for the APL effect in the Earth fixed coordinate system (dark curves). For comparison, the curves from Fig. 2 reflecting the variation of the GCC by the APL corrections are repeated (light

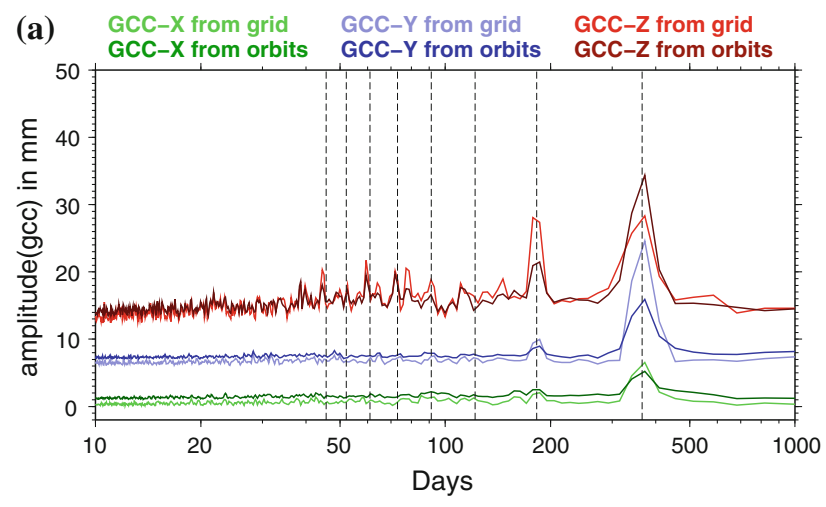

Fig. 15 Amplitude spectra of the results from Fig. 14. Individual components are shifted by $6 \mathrm{~mm}$. The dashed lines indicate the period of 1 year (365.25 days) and its harmonics. a Default orbit determination

The experiment performed in this section demonstrates that ignoring the APL during the GNSS data processing may affect the GNSS satellite orbits, depending on the orbit model and orbit parameter constraining. Even if the orbit model as it is applied at CODE seems robust for variations of the GCC generated by APL there are other ACs in the IGS that apply other or even no constraints to the once-per-revolution terms. Because the GNSS orbits cannot be corrected on the basis of weekly mean station coordinates (satellite orbits are not included in the weekly SINEX files of the IGS) these results are another important argument for applying the APL correction directly to the observations during the data processing.

\section{Summary}

The effect of APL is clearly visible in GNSS-derived coordinate time series. Because of the time variation in the APL effect a direct correction to the observations seems to be the right choice, in particular for weekly solutions. This direct

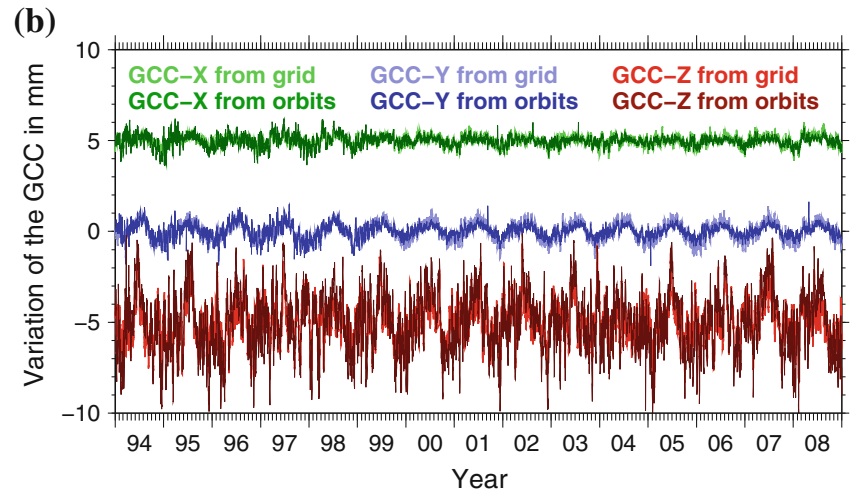

curves). Individual components are shifted by $5 \mathrm{~mm}$. a Default orbit determination scheme with constraints to the once-per-revolution terms. b Alternative orbit determination scheme without any constraints to the once-per-revolution terms

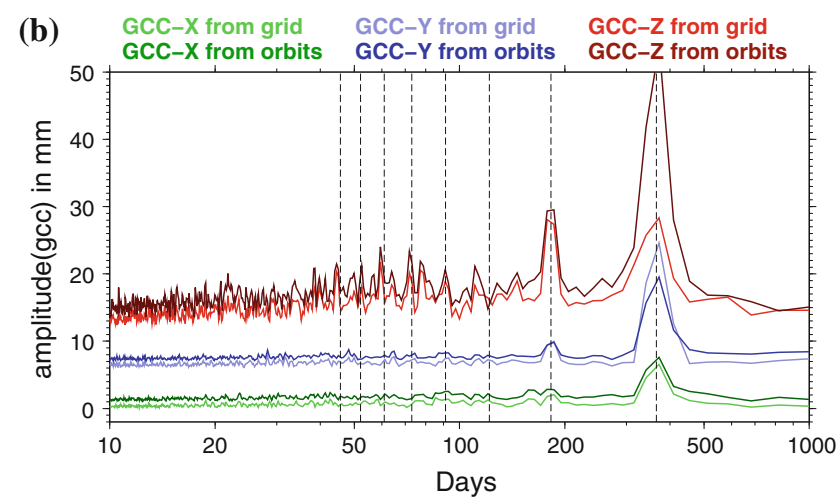

scheme with constraints to the once-per-revolution terms. b Alternative orbit determination scheme without any constraints to the once-per-revolution terms

correction is justified in particular because the real GNSS observation scenario (with data outages or the variation of the number of measurements per epoch, e.g., due to the satellite visibility) is not unambiguously recoverable from weekly or daily solutions exchanged between the analysis centers. In addition, once-per-revolution orbit parameters may also be affected by the APL effect depending on the used orbit model and the orbit parameter constraining. Information considering the APL corrections has to be documented for further use, e.g., for the combination of results (as it is done today, e.g., for correcting the ocean tidal loading effect).

The application of APL corrections on the observation level improves the repeatability of weekly station heights between 10 and $20 \%$ on the average. Correcting weekly solutions for APL only on the basis of weekly mean values from the same model reduces the gain roughly by a factor of two. The application of APL via regression factors between the time series of local pressure and vertical site displacement is also less beneficial than the application of APL on the observation level, because the distribution of the pressure in the 
vicinity of the station has a significant impact on the APL of the station and the effect in the horizontal components is completely ignored.

Because observation-level corrections for the APL effect cannot be removed from the solutions, the particular model needs to be carefully checked before making use of the corrections. For this purpose, scaling factors for the model of Petrov and Boy (2004) were directly estimated during the GNSS processing of the 15 years of data considered here. The Petrov and Boy (2004) model agrees within the expected uncertainty level of the model with the GNSS analysis. By stacking the scaling factors of the same months within the year(s) in the entire 15-year interval a seasonal variation of the scaling factors was derived. When comparing monthly regression factors between an APL model and the local pressure field the variation is much smaller. Other effects of the same order of magnitude as APL (e.g., ocean non-tidal or continental water mass surface load) and currently not taken into account in the GNSS analysis are most likely responsible for this result.

Acknowledgments The authors would like to thank the International GNSS Service (IGS Dow et al. 2009) for providing global GNSS observation data. The time series of displacements caused by the APL were provided by the Service of the Atmospheric Pressure Loading run at the NASA GSFC (http://gemini.gsfc.nasa.gov/aplo, Petrov and Boy 2004). The figures were generated with the GMT software (Generic Mapping Tools, Wessel and Smith 1998).

\section{References}

Altamimi Z, Collilieux X, Legrand J, Garayt B, Boucher C (2007) ITRF2005: a new release of the International Terrestrial Reference Frame based on time series of station positions and Earth orientation parameters. J Geophys Res 112(B9):401-419. doi:10.1029/2007JB004949

Beutler G, Brockmann E, Gurtner W, Hugentobler U, Mervart L, Rothacher M, Verdun A (1994) Extended orbit modeling techniques at the CODE processing center of the International GPS Service for Geodynamics (IGS): theory and initial results. Manuscr Geodaetica 19(6):367-386

Blewitt G (2003) Self-consistency in reference frames, geocenter definition, and surface loading of the solid earth. J Geophys Res 108(B2):2103. doi:10.1029/2002JB002082

Bock D, Noomen R, Scherneck HG (2005) Atmospheric pressure loading displacement of SLR stations. J Geodyn 39:247-266. doi:10. 1016/j.jog.2004.11.004

Böhm J, Niell A, Tregoning P, Schuh H (2006a) Global mapping function (GMF): a new empirical mapping function based on numerical weather model data. Geophys Res Lett 33:L07304. doi:10.1029/ 2005GL025546

Böhm J, Werl B, Schuh H (2006b) Troposphere mapping functions for GPS and VLBI from ECMWF operational analysis data. J Geophys Res 111:B02406. doi:10.1029/2005JB003629

Böhm J, Heinkelmann R, Schuh H (2007) Short note: a global model of pressure and temperature for geodetic applications. J Geod 81(10):679-683. doi:10.1007/s00190-007-0135-3
Böhm J, Heinkelmann R, Mendes Cerveira PJ, Schuh H (2009) Atmospheric loading corrections at the observation level in VLBI analysis. J Geod 83(11):1107-1113. doi:10.1007/s00190-009-0329-y

Collilieux X, Altamimi Z, Coulot D, van Dam T, Ray J (2010) Impact of loading effects on determination of the International Terrestrial Reference Frame. Adv Space Res 45(1):144-154. doi:10.1016/j. asr.2009.08.024

Dach R, Beutler G, Bock H, Fridez P, Gäde A, Hugentobler U, Jäggi A, Meindl M, Mervart L, Prange L, Schaer S, Springer T, Urschl C, Walser P (2007) Bernese GPS software version 5.0. Astronomical Institute, University of Bern, Switzerland

Dach R, Springer T, Altamimi Z (2008) Experiment on impact of constrained orbit parameters on station coordinates. In: International GNSS Service: Analysis Center Workshop. Miami Beach, Florida, USA

Dow J, Neilan R, Rizos C (2009) The International GNSS Service in a changing landscape of Global Navigation Satellite Systems. J Geod 83(3-4):191-198. doi:10.1007/s00190-008-0300-3

Farrell WE (1972) Deformation of the Earth by surface loads. Rev Geophys Space Phys 10:761-797. doi:10.1029/RG010i003p00761

Ferland R, Piraszewski M (2009) The IGS-combined station coordinates, Earth rotation parameters and apparent geocenter. J Geod 83(3-4):385-392. doi:10.1007/s00190-008-0295-9

Kaniuth K, Vetter S (2006) Estimating atmospheric pressure loading regression coefficients from GPS observations. GPS Solut 10(2):126-134. doi:10.007/s10291-005-0014-4

Kouba J (2008) Implementation and testing of the gridded Vienna Mapping Function 1 (VMF1). J Geod 82(4-5):193-205. doi:10.1007/ s00190-007-0170-0

MacMillan DS, Gipson JM (1994) Atmospheric pressure loading parameters from very long baseline interferometry observations. J Geophys Res 99(B9):18,081-18,087. doi:10.1029/94JB01190

Manabe S, Sato T, Sakai S, Yokoyama K (1991) Atmospheric loading effect on VLBI observations. In: AGU Chapman conference on geodetic VLBI: monitoring global change. NOAA technical report NOS 137, NGS 49, U.S. Department of Commerce, NOAA/NOS, Rockville, MD, pp 111-122

McCarthy D, Petit G (2004) IERS Conventions (2003). IERS technical note 32. Bundesamt für Kartographie und Geodäsie, Frankfurt am Main

Ostini L, Dach R, Meindl M, Schaer S, Hugentobler U (2008) FODITS: a new tool of the Bernese GPS software. In: Ihde J, Hornik H (eds) EUREF symposium, no. 18, June 18-20, 2008, Brussels, Belgium. EUREF Publication (in print)

Petrov L, Boy JP (2004) Study of the atmospheric pressure loading signal in very long baseline interferometry observations. J Geophys Res 109:B03405. doi:10.1029/2003JB002500

Rabbel W, Zschau J (1985) Static deformations and gravity changes at the Earth's surface due to atmospheric loading. J Geophys 56:8199

Schaer S, Dach R, Meindl M (2008) CODE analysis strategy summary. http://www.aiub.unibe.ch/download/CODE/CODE.ACN

Schmid R, Steigenberger P, Gendt G, Ge M, Rothacher M (2007) Generation of a consistent absolute phase center correction model for GPS receiver and satellite antennas. J Geod 81(12):781-798. doi:10.1007/s00190-007-0148-y

Steigenberger P, Böhm J, Tesmer V (2009a) Comparison of GMF/GPT with VMF1/ECMWF and implications for atmospheric loading. J Geod 83(10):943-951. doi:10.1007/s00190-009-0311-8

Steigenberger P, Schaer S, Lutz S, Dach R, Ostini L, Hugentobler U, Bock H, Jäggi A, Meindl M, Thaller D (2009b) CODE contribution to IGS reprocessing: status and perspectives. EGU General Assembly, Vienna, Austria 19-24

Tesmer V, Böhm J, Meisel B, Rothacher M, Steigenberger P (2008) Atmospheric loading coefficients determined from homogeneously reprocessed GPS and VLBI height time series. In: 
Finkelstein A, Behrend D (eds) Measuring the future. Proceedings of the 5th IVS general meeting, pp 307-313

Tregoning P, van Dam T (2005) Atmospheric pressure loading corrections applied to GPS data at the observation level. Geophys Res Lett 32:L22310. doi:10.1029/2005GL024104

Tregoning P, Watson C (2009) Atmospheric effects and spurious signals in GPS analyses. J Geophys Res 114:B09403. doi:10.1029/ 2009JB006344

van Dam T, Herring TA (1994) Detection of atmospheric pressure loading using Very Long Baseline Interferometry measurements. J Geophys Res 99(B3):4505-4517. doi:10.1029/93JB02658

van Dam T, Wahr J, Milly PCD, Shmakin AB, Blewitt G, Lavalee D, Larson K (2001) Crustal displacements due to continental water loading. Geophys Res Lett 28(4):651-654. doi:10.1029/ 2000GL012120 van Dam T, Plag HP, Francis O, Gegout P (2003) GGFC special bureau for loading: current status and plans. In: Richter B, Schwegmann W, Dick WR (eds) Proceedings of the IERS workshop on combination research and global geophysical fluids. IERS technical note, no. 30. Verlag des Bundesamts für Kartographie und Geodäsie, Frankfurt am Main, pp 180-198

Wessel P, Smith WHF (1998) New, improved version of Generic Mapping Tools released. EOS Trans Am Geophys Union 79(47):579

Zumberge JF, Heflin MB, Jefferson DC, Watkins MM, Webb FH (1994) Precise point positioning for the efficient and robust analysis of GPS data from large networks. J Geophys Res 102(B3):5005-5017. doi:10.1029/96JB03860 